\title{
LA ATENCIÓN PSICOPEDAGÓGICA DEL MAESTRO A LAS DIFICULTADES DEL APRENDIZAJE EN ESCOLARES PRIMARIOS EN EL CONTEXTO ANGOLANO
}

\section{Psychopedagogical attention of the teacher to the difficulties of learning in primary schools in the Angolan context}

\begin{abstract}
Evarista Joaquina Daniel Tchimuco, Lic. Universidad Estatal de Angola, Angola https://orcid.org/0000-0002-3208-3088 evaristadanielj@gmail.com
\end{abstract}

\author{
Juana María Cúbela González, Dr. C. \\ Universidad de Oriente, Cuba \\ https://orcid.org/0000-0002-1585-6536 \\ jmcubela@uo.edu.cu
}

\author{
Martha Martínez Isaac, Dr. C. \\ Universidad de Oriente, Cuba \\ https://orcid.org/0000-0002-7718-1052 \\ jmcubela@uo.edu.cu
}

Palabras claves: Proceso de Enseñanza Aprendizaje, Dificultades en el Aprendizaje, Atención Psicopedagógica.

Recibido: 18 de diciembre de 2018

Keywords: Teaching-Learning Process, Difficulties in Learning, Psycho-Pedagogical Attention.

Aceptado: 25 de marzo de 2019

\section{RESUMEN}

El presente artículo expone un tema de gran actualidad y pertinencia dentro de la sociedad en general y en particular en Angola, relacionada con el proceso de enseñanza-aprendizaje en la escuela primaria, y refleja cómo se trabaja en el perfeccionamiento constante dentro del sistema nacional de educación. Se reflexiona sobre las potencialidades de la atención psicopedagógica, como alternativa para el mejoramiento este importante proceso. Se brinda al maestro algunas premisas psicopedagógicas para la dirección efectiva del proceso en los escolares con dificultades en el aprendizaje.

\begin{abstract}
This article presents a topic of great relevance and relevance within society in general and in Angola in particular, related to the teaching-learning process in primary school, and reflects how work is constantly being done within the national education system. education. We reflect on the potential of psych pedagogical attention, as an alternative to improve this important process. The teacher is offered some psycho-pedagogical premises for the effective management of the process in schoolchildren with learning difficulties
\end{abstract}




\section{INTRODUCCIÓN}

La educación en Angola constituye un eslabón fundamental en el desarrollo científico y cultural del país y es una prioridad para el gobierno, en aras de formar una generación mejor preparada. En esta investigación se aborda el proceso de enseñanza- aprendizaje y especialmente las dificultades del aprendizaje de los escolares de primaria en Angola.

Para la República de Angola tiene una significativa importancia los diferentes problemas sociales y económicos que influyen en la educación de sus ciudadanos.

En la sociedad angolana se encuentran todavía problemas sociales, educativos, económicos y culturales, entre otros que repercuten en el sistema educativo de forma general. Muchos de estos problemas son consecuencia de largos años de explotación colonial por lo que todavía tiene atrasos significativos en el dominio educativo.

En el año 2009 fue considerado, dentro del África Subsahariana, uno de los países con elevada tasa de analfabetismo literal y de baja escolarización, lo que condiciona negativamente el proceso de recuperación y estabilidad socioeconómica del país. Unesco, (2004).

Por otra parte, la situación de la educación general y en particular, la enseñanza primaria en la República de Angola muestra deficiencias en la preparación de los maestros para dirigir eficientemente el proceso de enseñanza aprendizaje de los escolares, lo que devela la imperiosa necesidad de atender el desarrollo exitoso del aprendizaje de los escolares.

En la Ley de Bases del Sistema de Educación de Angola del 31 de diciembre de 2001, se plantea la necesidad de desarrollar armoniosamente las capacidades físicas, intelectuales, morales, cívicas, estéticas y laborales de la joven generación, de manera continua y sistemática y elevar su nivel científico, técnico y tecnológico, con el fin de contribuir al desarrollo socioeconómico del país.

En la mencionada ley también se hace alusión a la necesidad de formar un individuo capaz de comprender los problemas nacionales, regionales e internacionales de forma crítica y constructiva para su participación activa en la vida social, a la luz de los principios democráticos que se enarbolan en el país. Lo anteriormente expuesto, demuestra la necesidad de que la escuela primaria angolana actual tome como base las mejores experiencias de la práctica escolar emanadas de las múltiples investigaciones realizadas en el orden psicopedagógico en el país y en otros países, desde la propia práctica educativa, con la participación de maestros, directivos, escolares y padres.

Las transformaciones que se producen en la escuela actual exigen remodelar su funcionamiento para que cumpla con mayor calidad el papel socializador que le corresponde en la formación de la personalidad de las nuevas generaciones, donde el maestro desempeña un importante papel como orientador de las actividades y relaciones que tienen lugar en este contexto para lograr que los escolares aprendan mejor de manera que ellos se conviertan en agentes de su propio aprendizaje.

Cada vez más se les exige a los maestros que enseñen a sus escolares a aprender y a saber cómo aprenden, a cómo desarrollar mejor su pensamiento y la capacidad de aplicar lo aprendido a nuevas situaciones, con lo cual estarían en condiciones de transformarse a sí mismo y transformar la realidad, es por ello, que el futuro los escolares depende por entero de lo que sean capaces de enseñar los maestros desde la escuela primaria.

Entre los objetivos específicos de la enseñanza primaria se destacan el desarrollar y perfeccionar el dominio de la comunicación y la expresión, así como perfeccionar hábitos y actitudes para la socialización, proporcionar conocimientos y capacidades de desarrollo de las facultades mentales, estimular la creación artística, y la educación física entre otras. Su cumplimento garantizará la formación integral de la personalidad del escolar, fomentando desde los primeros grados, la interiorización de conocimientos y orientaciones valorativas que se reflejen gradualmente en sentimientos, formas de pensar y comportamiento adecuados.

A pesar de lo planteado el perfeccionamiento del proceso de enseñanza-aprendizaje en Angola se ha convertido en los últimos años en centro de atención de investigadores, pedagogos, psicólogos y sociólogos, dada la necesidad fundamentada por los avances científico-técnicos alcanzados y por el reconocimiento de la responsabilidad de la escuela con la formación de profesionales bien y ampliamente preparados desde el punto de vista metodológico, pedagógico, psicológico y sociológico.

Particularmente, la atención especializada del maestro a los escolares desde los primeros grados debe estar orientada a lograr progresivamente la formación de una personalidad apta para saber aprender y desarrollar sentimientos nobles y voluntades, lo cual no se limita a la apropiación de los fundamentos de las diferentes asignaturas del currículo, sino, 
también a potenciar el conocimiento de sí mismo, su autovaloración, confianza, autoestima, cultivo de un pensamiento reflexivo y crítico, capacidad para enfrentar los problemas del conocimiento y de la vida práctica, para tomar decisiones y desarrollar formas de hacer interesantes e inteligentes, proyectando su propia disposición a ser mejores seres humanos para bien personal y de los demás.

Lo expresado está en correspondencia con la necesaria preparación que debe alcanzar el maestro para lograrlo, y de disponer de las herramientas que estimulen las vías para contribuir al alcance de los pilares básicos de la educación Delors, J. (1996).

Aprender a conocer: Dada la rapidez provocada por los progresos científicos y las nuevas formas de actividad económica-social, es menester, promover, no solo el acceso a la información, sino la curiosidad y el deseo de conocer de forma permanente.

Aprender a hacer: Adquirir competencias que permitan hacer frente a las nuevas situaciones y que faciliten el trabajo en equipo, dimensión que tiende a disminuirse con los actuales métodos de enseñanza.

Aprender a ser: Esta premisa defiende que el progreso de las sociedades, depende de la creatividad y la capacidad de innovación de cada individuo.

Aprender a vivir juntos: Aspira a desarrollar el conocimiento de los demás, de su historia, tradiciones y su espiritualidad, en sociedades cada vez más multiculturales y competitivas.

Enseñar a los escolares a aprender, a conocer, a hacer, a ser y a vivir juntos significa a decir de Castellanos, D (1999) superar las concepciones de la enseñanza primaria, centrada en el maestro como eje básico y omnipotente de un proceso eminentemente instructivo, guardián-trasmisor de un conocimiento supuestamente acabado, incuestionable e imperecedero, requiere de una reconceptualización previa del proceso de aprendizaje.

La manera en que se proyecte y desarrolle el proceso de enseñanza aprendizaje en la escuela primaria debe intencionar la atención psicopedagógica a todos y cada uno de los escolares, en especial aquellos que presentan dificultades en el aprendizaje para incorporarlos activamente en la formación de su personalidad y del proceso de su desarrollo y autodesarrollo.

La investigación que se realiza posee una gran significación teórica y práctica en tanto se brindan premisas y pautas psicopedagógicas al maestro para favorecer el aprendizaje de los escolares de cuarto grado en el colegio 51 de la provincia de Huila- Angola, en función de promover la comunicación afectiva, la colaboración grupal y la atención psicopedagógica individualizada a las dificultades y a mejorar el proceso de aprendizaje de estos.

De ahí que se declare como objetivo: reflexionar en torno a los fundamentos epistemológicos del proceso de enseñanza aprendizaje en la escuela primaria, con énfasis en los escolares con dificultades en el aprendizaje, en especial de los escolares que se investigan de cuarto grado en el colegio 51 de la provincia de Huila-Angola.

\section{DESARROLLO}

El proceso de enseñanza-aprendizaje en la escuela primaria en el contexto angolano: una reflexión necesaria

El presidente José Eduardo dos Santos expresó (2008) es importante que los actuales avances políticos, económicos e institucionales del país se revelen en el plano social y en el plano de cambio de mentalidades, es un marco importante para el recate de una nueva mentalidad en la sociedad angoleña de modo que se propicie el desarrollo del conocimiento científico, el cual se realiza por medio de una educación integral".

En su intervención se destaca como elemento primordial a la educación que propicie avances significativos en el sector educacional del país como situación fundamental e indispensable para la dignidad plena de los ciudadanos. Por lo que, en la actualidad en Angola, a pesar de la voluntad política del gobierno, todavía la práctica educativa no logra satisfacer las exigencias del Estado y las expectativas de la familia, dado en lo fundamental, por insuficiencias que aún se presentan en la dirección del proceso de enseñanza-aprendizaje

La calidad de la educación es un problema de gran actualidad a nivel internacional y nacional, constituye una premisa de la política educativa en los diferentes niveles de enseñanza de la República de Angola. En la Ley de Bases del Sistema de Educación de Angola del 31 de diciembre de 2001, se plantea la necesidad de desarrollar armoniosamente las capacidades físicas, intelectuales, morales, cívicas, estéticas y laborales de la joven generación, de manera continua y sistemática y elevar su nivel científico, técnico y tecnológico, con el fin de contribuir al desarrollo socioeconómico del país. 
Se hace alusión a la necesidad de formar un individuo capaz de comprender los problemas nacionales, regionales e internacionales de forma crítica y constructiva para su participación activa en la vida social, a la luz de los principios democráticos que se elevan en el país.

En Angola, y en el mundo, el proceso de enseñanza aprendizaje, ha sido visto de diferentes maneras desde el siglo XVI hasta nuestros días. En su devenir histórico, prestigiosos educadores han realizado aportacionespor la importancia de este proceso, han constituido herramientas para la estructuración del proceso de enseñanzaaprendizaje en su enfoque sistémico y sistemático.

De igual forma, se asumen las principales concepciones y fundamentos sobre el proceso de enseñanza aprendizaje, siendo los más recurrentes: Vigostky, S. (1978); Labarrere, G. (1988);Torres, M. (1990); Vlasova, T. A. (1992); Morenza, L. (1996); García Sánchez, (1998); Castellanos (2001-2002); Rodríguez, A. (2005); Silvestre y Zilberstein, (2002); Mestre, U.(2010); Addine, F. (1998,2004,2013); Betancourt,J. y González, A. Olga (2003); Soca, A.M.(2004); Rico,P.(2008);Silvestre, M.(2000); Hurtado, J. (2003), Zayas, C. (1996).

De manera general, los autores referenciados han demostrado que durante años los estudios pedagógicos, psicológicos y metodológicos han centrado su atención en cómo transcurre el proceso de enseñanza, qué acciones realiza el profesor para poder enseñar los contenidos de una asignatura o una disciplina dada, cómo este prepara su clase, cómo se auto prepara, cómo dirige el proceso de enseñanza, y en menor medida, cómo ocurre el aprendizaje en correspondencia con dicho proceso.

El proceso de enseñanza-aprendizaje, sin lugar a dudas, lleva implícito dos procesos inseparables y bilateralmente dialécticos que se dan en una unidad e interrelación y condicionan mutuamente: enseñanza y aprendizaje. El proceso de enseñanza responde básicamente a la actividad del docente y el proceso de aprendizaje, en tanto depende del anterior, se lleva a cabo por los alumnos, dirigidos por el profesor.

Los referentes valorados anteriormente dan respuesta a las exigencias actuales de la escuela primaria angolana y a la necesidad de perfeccionar el aprendizaje de los escolares con dificultades permitiendo que ellos sean capaces de:

- Ordenar, clasificar y jerarquizar conceptos.

- Integrar los contenidos desarrollados durante una unidad o subunidad de cualquiera de los programas de un grado o nivel, para resolver problemas que exijan niveles de integración y generalización en su solución.

- Confrontar, modificar y enriquecer el conocimiento práctico y teórico que poseen los escolares.

- Evidenciar la articulación entre la teoría y la práctica.

- Favorecer el trabajo colaborativo, en grupos.

La autora considera que el aprendizaje es un proceso en que el escolar, debe ser estimulado siempre por el maestro, para adoptar una actitud protagónica y activa, lo que le permitirá una mejor apropiación de conocimientos, habilidades y capacidades, valores en comunicación con los otros, a través de un proceso de socialización que se va haciendo cada vez más significativo para él.

De modo que, se asume que el aprendizaje es un proceso complejo, diversificado, altamente condicionado por las características del sujeto, las situaciones y contextos socioculturales en que se aprende, los contenidos de enseñanza, los recursos con que cuenta para ello, el nivel de intencionalidad, la organización, entre otras condiciones educativas. (Castellanos, 1998).

Desde esta perspectiva lo histórico-cultural está presente desde concepción desarrolladora del aprendizaje, significando algunos rasgos esenciales:

- Aprender es un proceso que ocurre a lo largo de toda la vida.

- En el aprendizaje cristaliza continuamente la dialéctica entre lo histórico-social y lo individual personal.

- Aprender supone un tránsito de lo externo a lo interno parafraseando a Vigotsky, de lo interpsicológico a lo intrapsicológico.

- El proceso de aprendizaje posee tanto un carácter intelectual como emocional.

- Aprender es un proceso individual, que requiere participación, colaboración e interacción.

- El aprendizaje es multidimensional por sus contenidos, procesos y condiciones.

- Los contenidos del aprendizaje llevan el signo de la diversidad.

- Se aprende en la actividad y la comunicación.

\section{La atención a los escolares con dificultades en el aprendizaje: una necesidad y un reto en la escuela actual}

En consonancia con lo que se ha expresado, la enseñanza primaria en Angola, enfrenta en la actualidad una serie de transformaciones que constituyen condiciones favorables para conducir un proceso educativo con mayor calidad, 
influenciado fundamentalmente por un reducido número de matrícula de alumnos por aula y por la inserción de la tecnología, este último complemento significativo para los procesos educativos que se desarrollan en la misma.

La definición de dificultades de aprendizaje ha sido un verdadero problema en el que se han concentrado numerosos especialistas, los que desde diversas posiciones teóricos-metodológicas han ofrecido diferentes aproximaciones en las que no hay aún una comprensión y delimitación que exprese un total consenso de criterios Kirk, S. (1963); Hammill, (1990); González A. (1993); Torres, M. (2003), entre otros.

Al abordar las dificultades en el aprendizaje, de manera general, los autores refieren tres planos básicos que guardan relación con las posibles causas que la generan: el pedagógico donde se aprecian dificultades en todas las materias escolares; el psicológico, dificultades en los procesos psíquicos y el neurológico en casos de disfunción en el Sistema Nervioso Central (SNC).

Para Hammill (1990) las dificultades de aprendizaje son un término genérico que se refiere a un grupo heterogéneo de trastornos debidos a disfunciones en el sistema nervioso central sea de forma identificable o inferida. Tales trastornos pueden manifestarse porretrasos en el desarrollo precoz y/o dificultades en alguna de las siguientes áreas: atención, memoria, razonamiento, coordinación, comunicación, lectura, escritura, deletreo, cálculo, competencia social y maduración emocional.

La autora asume la definición de dificultades en el aprendizaje que aparece en el libro Selección de lecturas sobre Retardo en el Desarrollo Psíquico, de Torres M. (2003), en el que se establece que es la dificultad para apropiarse de los contenidos básicos de los materiales escolares y para utilizar procedimientos adecuados en la solución de las tareas docentes fundamentadas por insuficiente desarrollo u organización de los procesos psíquicos que intervienen en el proceso de aprendizaje.

Las dificultades de aprendizaje son intrínsecas y pueden afectar al aprendizaje y la conducta en algunos escolares, incluyendo a los de inteligencia potencialmente normal o superior. Las mismas no se deben, primariamente, a minusvalías visuales, auditivas o motrices, al retraso mental, trastorno emocional o desventaja cultural, aunque puede ocurrir concurrentemente con cualquiera de ellas.

Se puede decir que las dificultades de aprendizaje son un término genérico, que designa un conjunto heterogéneo de desórdenes, manifestado en dificultades significativas en la adquisición y uso de las capacidades comprensión oral, lectura, escritura, razonamiento y para la matemática.

Estos desórdenes son intrínsecos al individuo, presumiblemente, debidos a una disfunción del (SNC) y pueden ocurrir a lo largo de toda la vida. Los problemas en comportamientos que requieren autocontrol, percepción e interacciones sociales pueden coexistir con las dificultades en el aprendizaje, pero no constituyen en sí mismo una dificultad en el aprendizaje.

\section{La atención psicopedagógica: premisas al maestro para la dirección de proceso de enseñanza aprendizaje de los escolares en el contexto angolano}

La atención psicopedagógica ha sido abordada desde diversas ciencias y múltiples referencias.

Existe coincidencia ente los autores en considerarla como un proceso para dar respuesta a las diferencias individuales y socioculturales, a través del desarrollo de acciones integrales en igualdad de oportunidades y equiparación de condiciones en todas las instituciones educativas regulares, mediante la implementación de adaptaciones curriculares, ayuda profesional sistemática de maestros, apoyo de profesionales, trabajo con padres de familia, comunidad y sociedad.

Específicamente, al abordar la atención psicopedagógica de los escolares se asumen las consideraciones de Cubela G, J.M.(2017), la que refiere esta atención tiene que ver con la labor o el trabajo orientado a todos los escolares que necesitan de ayuda profesional en alguna o diversas áreas de su desarrollo personal, social y escolar, siendo necesario crear un clima socio afectivo estimulador de seguridad, animación y confianza entre los maestros y escolares, que permita profundizar en aspectos de su personalidad en formación e influir de manera directa o indirectamente en su desempeño.

Así como identificar y canalizar sus principales problemas y potencialidades para ofrecer el apoyo pedagógico y psicológico oportuno, señalar y sugerir actividades docentes, extra docentes y extracurriculares que favorezcan su desarrollo integral. 
Para que la atención psicopedagógica fluya el maestro no solamente debe poner en práctica un sistema de conocimientos, de principios y leyes generales de la enseñanza y el aprendizaje, sino también ser capaces de integrar en el proceso las recomendaciones médicas, psicológicas y de otras especialidades.

Para cada caso en particular, y estimular el aprendizaje de sus escolares utilizando vías específicas de influencia psicopedagógica que propicien la corrección de los procesos y cualidades afectivas y cognoscitivas más dañadas en estos escolares.

Por consiguiente, la atención psicopedagógica tiene como propósito atender a los escolares que presentan dificultades en el proceso de enseñanza aprendizaje, sus actitudes, emociones, afectividad, voluntad, etc. y que de alguna manera interfieren en su adecuado desempeño escolar.

Este requiere atender de manera oportuna las necesidades psicopedagógicas de los alumnos y estimular sus potencialidades de desarrollo personal, mediante el trabajo intencionado del maestro en colaboración con la familia, el grupo y otros agentes socializadores que se relacionen con estos, mediante asesorías pedagógicas y psicológicas que contribuyan a estimular y desarrollar su proceso formativo.

Por tanto, el apoyo psicopedagógico del maestro al escolar es necesario durante su trayectoria escolar y su proceso formativo en el ámbito de:

$>$ La construcción de valores,

$>$ Actitudes,

$>$ Hábitos positivos y

$>$ La promoción del desarrollo de habilidades intelectuales.

Llevando a cabo un proceso de atención personalizada que complementen las actividades docentes regulares a fin de que cuenten con mayores oportunidades para su éxito académico y en el aprendizaje, así como dar seguimiento al progreso de su evolución social y personal.

Se enfatiza en que el maestro debe poseer los conocimientos y los recursos psicopedagógicos necesarios para aportar información primaria sobre la atención personalizada que pueden realizar sobre los problemas personales que poseen los escolares, con vista a atender aquellas situaciones que pueden incidir en su aprendizaje y en su vida personal.

En este caso, se debe priorizar la familia que requiere de la orientación del maestro para poder mancomunadamente con este dar las ayudas que sus hijos necesitan. De manera que el proceso de atención psicopedagógica personalizada del maestro debe estar dirigido a movilizar todo el potencial cognitivo, afectivo, motivacional y autorregulador de los escolares, a favor del desarrollo de sus procesos cognitivos, afectivos-motivacionales, habilidades, capacidades, a través de un proceso de construcción y reconstrucción de su equilibrio, orientado al alcance de niveles superiores de desarrollo, a un crecimiento en relación con los objetivos que demanda el modelo de la escuela primaria en concreto al grado que cursa.

El maestro puede desarrollar varias acciones y utilizar herramientas para atender psicopedagógicamente los escolares de cuarto grado con dificultades de aprendizaje, entre ella tenemos:

- Incluirlos en grupos no muy grandes, ubicándolos como parte del grupo e integrarlos a la situación de aprendizaje.

- Proporcionarles tareas subdivididas.

- Permitirles salir, por períodos cortos, del aula con alguna consigna o encomienda específica.

- Destacarles sus logros, que, aunque sean pequeños en comparación a los de sus compañeros, son grandes para ellos.

- Otorgándoles un marco de contención.

- Tener una comunicación periódica con los padres, a los que se les debe informar tanto de las dificultades como los logros.

- Respetarles sus tiempos.

- Revisar qué es lo más relevante del programa y plantearles metas posibles.

- Posibilitar el trabajo en un equipo, que acepte que no existe un saber totalizador, ni recetas rápidas y mágicas que funcionen en todas las situaciones, tratándose, en numerosas ocasiones, de acompañar los desbordes de estos niños, mientras construyen su propio borde.

- Ayudarlos a organizar sus tareas.

- Mirarlos cuando se da una explicación o se realiza un relato, acompañar las palabras con acciones o gestos. 
También para atender los escolares con problemas de hay que plantearse cómo hacer que los escolares conecten el nuevo material que están aprendiendo con sus marcos de referencia y sistemas de categorías, a fin de hacerlos propios, y luego utilizar la información en forma compatible con la que ya conocen.

La motivación a los escolares resulta notable para que puedan sentirse estimulados a desplegar sus capacidades en la resolución de situaciones problemáticas y se sientan recompensados por su esfuerzo en la búsqueda de soluciones donde el desempeño correcto deberá actuar como una autorecompensa. Da la posibilidad de poner en práctica, en diferentes oportunidades, y las habilidades que van adquiriendo.

Específicamente, para la atención psicopedagógica de las dificultades en el aprendizaje el maestro debe atender los siguientes requerimientos:

- La evaluación psicopedagógica del escolar: A través del diagnóstico donde se tenga en cuenta la aplicación de pruebas de evaluación psicopedagógica que permitan determinar qué es lo que está interfiriendo en el aprendizaje del niño para que no se esté dando un aprendizaje eficaz.

- El entrenamiento de la atención sostenida y selectiva: Muchos de los problemas de aprendizaje tienen que ver con una escasa capacidad para mantener la atención durante un tiempo adecuado en una tarea y conseguir responder a los estímulos relevantes de la misma.

Ambas capacidades atencionales son fundamentales para el aprendizaje y se pueden entrenar.

- La aplicación de estrategias para el manejo de la impulsividad: En muchos casos, los escolares cometen fallos en sus tareas a pesar de la buena comprensión de las mismas porque la impulsividad les puede llevar a actuar sin que medie una reflexión que guíe su respuesta.

- La estimulación del razonamiento abstracto: El razonamiento abstracto es la facultad que permite analizar, extraer conclusiones y establecer conexiones causales y lógicas.

Cuando esta habilidad es deficiente en el escolar, hace más difícil su aprendizaje, pero es posible estimular ésta para mejorarla y conseguir así una mayor capacidad de aprendizaje.

- Apoyo en el aprendizaje del lector escritura: No todos los niños aprenden al mismo ritmo y hay algunos que van más lentos en dicho aprendizaje y necesitan una ayuda extra. Conviene también evaluar si se encuentran ante una dislexia.

En cualquier caso, dada la importancia de la lectoescritura en los futuros aprendizajes del niño, es importante intervenir cuando encontramos dificultades en esta área.

- Intervención para la mejora de la velocidad y comprensión lectora: La lectura silábica reduce la velocidad de lectura; esto perjudica la comprensión de lo que se está leyendo.

Por ello, es importante entrenar la velocidad lectora, potenciando la ruta visual de la lectura.

- La instrucción en la resolución de problemas matemáticos: La resolución de los problemas matemáticos es uno de los grandes desafíos para los escolares de primaria.

Muchos de ellos aún no tienen un razonamiento abstracto bien desarrollado y basan sus aprendizajes en el razonamiento concreto.

Conociendo esto, se trata de instruirles en un método de resolución de los problemas que se adapta a su capacidad de comprensión y les facilita plantear y desarrollar la resolución de los problemas matemáticos.

- Enseñar técnicas de estudio: Dedicar muchas horas al estudio no asegura el éxito.

La intervención del maestro debe estar enfocada a conseguir un estudio eficaz, que incluye una adecuada planificación, aprender a realizar un buen subrayado, una correcta esquematización de los conceptos que permita la comprensión y asimilación de los mismos, la memorización, los repasos.

Así, se puede ayudar al escolar, según el grado que transite, técnicas para que lleve a cabo un aprendizaje.

- Enseñar al escolar a saber comunicarse con los otros: a escuchar, a meditar antes de responder, a respetar las opiniones ajenas, a aprender de sus errores sin tener miedo a responder ante su grupo, etc.

- Estimular los sentimientos de afecto a sus compañeros: de colaboración entre equipos y subgrupos, de ayuda mutua, de priorizar el apoyo al más necesitado, así como la responsabilidad y actitud positiva ante la realización de tareas, la motivación por la participación en las diferentes actividades escolares, extraescolares y extra docentes.

- Brindar el apoyo necesario para desarrollar la autorregulación: la autoestima, la autovaloración adecuada de los niños y la seguridad propia de que pueden hacer buenas prácticas de aprendizaje. 
La efectividad del proceso de aprendizaje está influenciada por múltiples variables que inciden positiva o negativamente.

El maestro, como mediador y gestor de buenas prácticas de aprendizaje debe no sólo estar atento a esas variables, sino también conocerlas y asumirlas sistemáticamente en su práctica pedagógica. Una inquietud constante que suelen tener los maestros es cómo generar una enseñanza más efectiva que genere más y mejores aprendizajes por parte de los escolares.

Aunque esa inquietud se puede abordar desde varios ángulos, dependiendo de las necesidades de los escolares y de la preparación profesional del docente, es innegable la importancia de un diagnostico optimista, fino y profundo de las causas que inciden en las dificultades de sus educandos. Que permita un conocimiento más sólido de las diferencias individuales y una atención psicopedagógica oportuna, diferenciadora, sistemática y profesionalizada, lo que sin lugar a dudas puede generar aprendizajes más significativos y más efectivos en el alumnado.

\section{CONCLUSIÓN}

En las escuelas primarias en la República de Angola, aún muestran insatisfacciones en relación con la preparación de los maestros para dirigir eficientemente el proceso de enseñanza aprendizaje de los escolares, de ahí la necesidad de brindar una atención privilegiada en este aspecto en aras de elevar la calidad educativa.

La sistematización realizada permitió la elaboración de premisas al maestro para la dirección de proceso de enseñanza aprendizaje en los escolares con dificultades en el aprendizaje en el contexto angolano, a partir de priorizar la atención psicopedagógica.

La atención psicopedagógica personalizada, individual y sistemática de los maestros a los escolares que presentan dificultades en el aprendizaje, con la implementación de varias acciones de carácter psicopedagógico, herramientas y requerimientos metodológicos estimuladores de este proceso en cada escolar dentro de grupo, y con el apoyo de la familia en su sistematización, es un imperativo de la escuela angolana actualmente.

\section{BIBLIOGRAFÍA}

1. BERMÚDEZ (2004). La personalidad su diagnóstico y su desarrollo. La Habana: Editorial Pueblo y Educación.

2. BLANCO (2001). Introducción a la Sociología de la Educación. La Habana: Editorial Pueblo y Educación. ISPEJV.

3. CASTELLANO (2002). Aprender y Enseñar en la Escuela. Una concepción desarrolladora. La Habana: Editorial Pueblo y Educación.

4. CONSTITUCIÓN DE LA REPÚBLICA DE ANGOLA. (2001). Ley N. 13/01 de 31 de diciembre, artículo 88을 de la Ley Constitucional y Asamblea Nacional en Angola.

5. CUBELA G, J M. (2018). Caracterización, diagnóstico, orientación educativa y crecimiento personal: Orientaciones, vías, métodos, técnicas y ejemplificaciones para su realización. Editorial Academic Espanola is in cooperation with: MoreBooks! Marketing SRL Balti, 4 Industriala Street, Moldova, Europe. Link: https://www.eae-blishing.com/extern/listprojects.

6. CUBELA G, J M. (2017). Modelo pedagógico de la orientación educativa personalizada del maestro al escolar. Santiago de Cuba, 2

7. DOS SANTOS. (2008). Mensagen de Fin de Año. Jornal de Angola.

8. GARCÍA (2002). Compendio de Pedagogía. (Compilación). La Habana. Editorial Pueblo y Educación.

9. GONZÁLEZ (2004). La dinámica del proceso de enseñanza aprendizaje mediante sus componentes.

10. MESTRE (2010). Los eslabones del proceso docente educativo. Recuperado de: http://www.Bharatbhasha.com/education.php/17624.

11. RICO Y OTROS (2004). Proceso de enseñanza aprendizaje desarrollador en la Escuela Primaria. La Habana. Editorial Pueblo y Educación.

12. SILVESTRE, M. Y ZILBERSTEIN, J. (2001). Enseñanza y aprendizaje desarrollador. Instituto Central de Ciencias Pedagógicas, La Habana. (En soporte electrónico).

13. VYGOTSKY, L. S. (1983). Historia del desarrollo de las funciones psíquicas superiores. La Habana: Pueblo y Educación.

14. VYGOTSKY, L. S. (1998). Pensamiento y Lenguaje. La Habana: Pueblo y Educación. 